\title{
Impact of Resistance Training in Subjects With COPD: A Systematic Review and Meta-Analysis
}

\author{
Wen-hua Liao, Jin-wu Chen MM, Xin Chen MD, Lin Lin, Hai-yan Yan MM, Yu-qi Zhou MD, \\ and Rui Chen MD
}

\begin{abstract}
BACKGROUND: The goal of this study was to evaluate the effects of resistance training on subjects with COPD. METHODS: We performed a systematic search in MEDLINE, PubMed, Embase, CINAHL, Elsevier ScienceDirect, EBM Reviews, Cochrane Central Register of Controlled Trials, and ClinicalTrials.gov and also of leading respiratory journals for randomized controlled trials on COPD treatment for $\geq 4$ weeks with resistance training compared with non-exercise control or with combined resistance and endurance training compared with endurance training alone. Data from these studies were pooled to calculate odds ratio and weighted mean differences (WMDs) with 95\% CI. RESULTS: Eighteen trials with 750 subjects with advanced COPD met the inclusion criteria. There were 2 primary and 5 secondary outcomes. Compared with non-exercise control, resistance training led to significant improvements in the dyspnea domain of the Chronic Respiratory Disease Questionnaire (WMD of 0.59, 95\% CI 0.26-0.93, $\mathrm{I}^{2}=0 \%, P<.001$ ), skeletal muscle strength, and percent-of-predicted $\mathrm{FEV}_{1}$ (WMD of $6.88 \%, 95 \%$ CI $\left.0.41-13.35 \%, \mathrm{I}^{2}=0 \%, P=.04\right)$. The combination of resistance and endurance training significantly improved the St George Respiratory Questionnaire total score (WMD of $-7.44,95 \%$ CI -12.62 to $-2.25, \mathrm{I}^{2}=0 \%, P=.005$ ), each domain score, and skeletal muscle strength. There were no significant differences in 6-min walk distance, 6-min pegboard and ring test, maximum exercise work load, and maximum oxygen consumption between the 2 groups. There were no reports of adverse events related to resistancetraining intervention. CONCLUSIONS: Resistance training can be successfully performed alone or in conjunction with endurance training without increased adverse events during pulmonary rehabilitation in COPD. Key words: chronic obstructive pulmonary disease; resistance training; metaanalysis. [Respir Care 2015;60(8):1130-1145. (C) 2015 Daedalus Enterprises]
\end{abstract}

\section{Introduction}

COPD is a major cause of chronic morbidity and mortality throughout the world and is projected to be the third most common cause of death by $2020 .{ }^{1}$ Exercise intolerance is a cardinal complaint of patients with COPD. Skeletal muscle dysfunction is a common extrapulmonary man-

\footnotetext{
Ms Liao, Ms Lin, and Dr R Chen are affiliated with the Department of Respiratory Diseases, Mr Chen is affiliated with the Medical Examination Center, and Ms Yan is affiliated with the Department of Clinical Laboratory, Sun Yat-sen Memorial Hospital, Sun Yat-sen University, Guangzhou, China. Dr X Chen is affiliated with the Department of Respiratory Diseases, Zhujiang Hospital, Southern Medical University, Guangzhou, China. Dr Zhou is affiliated with the Department of Respiratory Diseases, The Third Affiliated Hospital, Sun Yat-sen University, Guangzhou, China.
}

Ms Liao, Mr Chen, and Dr X Chen are co-first authors. ifestation of COPD. ${ }^{2}$ Studies suggest that skeletal muscle dysfunction is associated with exercise limitation and health-care utilization. ${ }^{3,4}$ Skeletal muscle dysfunction is

\footnotetext{
This study was supported by Guangdong Provincial Science and Technology Project grant 2011B080701062 and Guangdong Natural Science Foundation grant S2012010008623. The authors have disclosed no conflicts of interest.

Correspondence: Rui Chen MD, Department of Respiratory Diseases, Sun Yat-sen Memorial Hospital, Sun Yat-sen University, 107 Yan Jiang Road, Guangzhou 510120, China. E-mail: gzchenrui@163.com. Yu-qi Zhou MD, Department of Respiratory Diseases, The Third Affiliated Hospital, Sun Yat-sen University, 600 Tian He Road, Guangzhou 510630, China. E-mail: zzyyqqcj7605@163.com.
}

DOI: $10.4187 /$ respcare. 03598 
also an independent predictor of morbidity and mortality in COPD, 5 irrespective of the degree of air-flow limitation. Muscle changes observed in patients with COPD include reductions in type I fibers, atrophy of type I and II fibers, reduced capillarity, and altered metabolic enzyme levels. ${ }^{6}$ The pathogenic mechanisms of skeletal muscle dysfunction are considered to be related to multiple factors, including nutritional abnormalities, muscle disuse, systemic inflammation, medical use of corticosteroids, tissue hypoxia, and hypercapnia. 6,7

Progressive resistance training provides a training modality for increasing peripheral muscle strength in COPD. Ortega et $\mathrm{al}^{8}$ reported that the increase in muscle strength obtained after resistance training is higher than that obtained after endurance training. In addition, resistance training evokes less dyspnea during exercise, ${ }^{9}$ thereby making this strategy easier to tolerate than endurance training. ${ }^{10,11} \mathrm{~A}$ combination of resistance and endurance training in COPD has demonstrated a greater improvement in peripheral muscle function compared with endurance training alone. ${ }^{12}$

In the past few years, there have been several systematic reviews on the efficacy of resistance training. ${ }^{13-16}$ However, previous meta-analyses focused on whether resistance training is effective in improving skeletal muscle strength and lung function, whereas little data are so far available on other clinically relevant outcomes, such as quality of life, dyspnea, and exercise capacity. Moreover, previous analyses ${ }^{13-15}$ included both randomized controlled trials (RCTs) and casecontrol trials, which potentially introduced bias because the real-world outcomes of pulmonary rehabilitation can be affected by a number of social and cultural factors. Finally, many RCTs have been published since the previous metaanalysis conducted by O'Shea et $\mathrm{al}^{14}$, offering input for more extensive analysis. ${ }^{17-23}$ The aim of this meta-analysis was to investigate the effects of resistance training alone or combined with endurance training on clinically relevant rehabilitation outcomes in advanced COPD, including quality of life, dyspnea, functional exercise capacity, maximum exercise capacity, skeletal muscle function, lung function, and adverse events.

\section{Methods}

\section{Data Sources}

We searched MEDLINE, PubMed, Embase, CINAHL, Elsevier ScienceDirect, EBM Reviews, Cochrane Central Register of Controlled Trials, ClinicalTrials.gov and leading respiratory journals and conference abstracts from January 1980 to October 2013 to identify related articles. We also searched the Science Citation Index database (Web of Science) and PubMed using the related-articles function by entering all included studies. Reference lists from original and review articles were also reviewed to identify additional relevant studies. All publications and abstracts

\section{QUICK LOOK}

\section{Current knowledge}

COPD is a major cause of chronic morbidity and mortality throughout the world and is projected to be the third most common cause of death by 2020. Skeletal muscle dysfunction is associated with exercise limitation and increased health-care utilization. The impact of respiratory muscle training in COPD has met with conflicting results.

\section{What this paper contributes to our knowledge}

A meta-analysis showed that dyspnea scale scores, skeletal muscle strength, and lung function improved following resistance training. Although skeletal muscle strength and quality of life improved following combined resistance and endurance training, this failed to translate into improved exercise capacity. The data suggest that resistance training can be successfully performed alone or in conjunction with endurance training without increasing adverse events during pulmonary rehabilitation.

in English were considered. Moreover, an additional search in May 2014 was performed to identify additional trials that fulfilled our search criteria.

The search terms were as follows: COPD, chronic obstructive pulmonary disease, chronic obstructive lung disease, chronic airways limitation, chronic airways obstruction, chronic bronchitis, and pulmonary emphysema. These terms were used in various combinations with strength training, strength exercise, resistance training, resistance exercise, weight training, weight lifting, aerobic training, aerobic exercise, endurance training, endurance exercise, exercise training, and pulmonary rehabilitation.

\section{Study Selection}

The inclusion criteria were: (1) subjects with stable moderate-to-very-severe COPD without other lung diseases; (2) RCTs comparing resistance training with non-exercise control or combined resistance and endurance training with endurance training alone; (3) exercise duration of at least 4 weeks; (4) outcomes including health-related quality of life, dyspnea scale, functional exercise capacity, maximum exercise capacity, skeletal muscle function, and pulmonary function; (5) human studies; and (6) English language.

\section{Quality Assessment}

The methodological quality of each study was assessed by the modified Jadad scale, ${ }^{24}$ which scores trials according to randomization, concealment of allocation, double blinding, withdrawals, and dropouts. 


\section{Data Extraction}

Data extraction was based on reported statistics (means, $\mathrm{SD}$, and SE). Two reviewers (WL and JC) independently extracted data from the selected studies. If a disagreement arose, all authors conferred until a consensus was achieved. Authors of a publication were contacted if only the abstract was available or data were missing. Supplemental data for included studies were reviewed to minimize selective reporting of secondary end points in published manuscripts. Primary outcomes were changes from baseline in health-related quality of life and dyspnea scale. Secondary outcomes included changes from baseline in skeletal muscle function, functional exercise capacity, maximum exercise capacity, $\mathrm{FEV}_{1}$, and adverse events.

\section{Statistical Analysis}

RevMan 5.2 (The Nordic Cochrane Centre, The Cochrane Collaboration, Copenhagen, Denmark) was used to analyze all collected data. Fixed-effects odds ratios for dichotomous outcomes and weighted mean differences (WMDs) for continuous outcomes, with corresponding 95\% CI, were calculated for individual trials. The trials were pooled using fixedeffects odds ratios or WMDs as appropriate. $\mathrm{I}^{2}$ was calculated to efficiently test heterogeneity, with values of 25,50 and $75 \%$ considered to represent low, moderate, and high heterogeneity, respectively. The differences between resistancetraining groups and non-exercise control groups or resistanceand-endurance-training groups and endurance-training-alone groups were pooled using a fixed-effects model when there was no evidence of significant heterogeneity in the analysis. If significant heterogeneity was found, a random-effects model was used. ${ }^{25}$

\section{Results}

\section{Search Results}

The process used for searching and selecting trials is presented in Figure 1. Of the 3,562 English articles screened, we excluded 3,544 that were not relevant, had incomplete or duplicate data, or were not RCTs. Eighteen parallel RCTs involving 750 subjects met the inclusion criteria and were selected for analysis. Thirteen of the 18 included trials compared resistance training with non-exercise control, and 4 trials compared combined resistance and endurance training with endurance training alone. One trial compared resistance training, endurance training, combined resistance and endurance training, and non-exercise control. The main characteristics of these trials are listed in Tables 1 and 2. All data adopted in this study were published openly in various journals.

\section{Quality Assessment}

The methodological quality of the included studies is provided in Table 2. There were 8 studies with Jadad

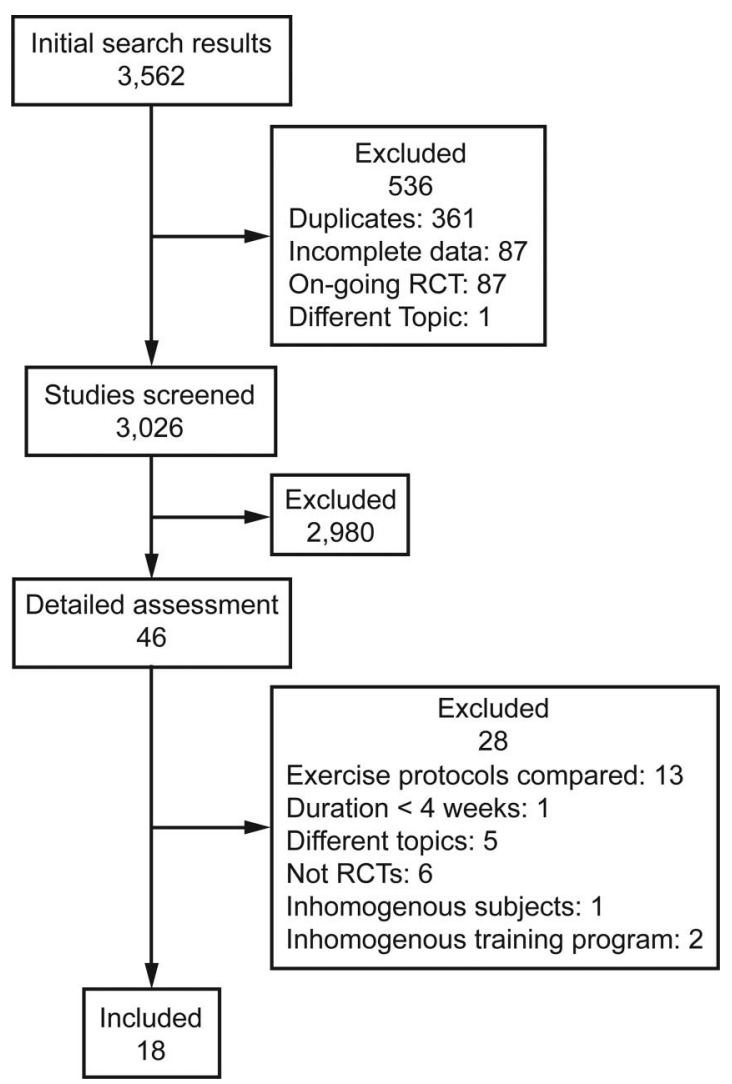

Fig. 1. Flow chart. RCT = randomized controlled trial.

scores of $\geq 3$ points. Ten trials scored poorly according to the modified Jadad scale. Eight trials reported blinding methods. Of these, 6 trials reported blinding of the investigators or outcome assessors, and 2 trials reported blinding of both outcome assessors and subjects with COPD. Per-protocol analysis was used in 16 trials, and intentionto-treat analysis was used in 2 trials.

\section{Primary Outcomes}

\section{Chronic Respiratory Disease Questionnaire Score}

Resistance-Training Group Versus Non-Exercise Control Group. Three studies reported dyspnea domain scores using the Chronic Respiratory Disease Questionnaire (CRQ). ${ }^{18,19,32}$ The results of each study showed significant improvements in CRQ dyspnea domain scores in the resistance-training groups. The overall analysis showed statistically significant improvements in CRQ dyspnea domain scores in the resistance-training groups (WMD of $0.59,95 \%$ CI $\left.0.26-0.93, \mathrm{I}^{2}=0 \%, P<.001\right)$. The improvement in dyspnea domain scores achieved a minimum clinically important difference of 0.5 units. ${ }^{37}$ Two included trials reported CRQ fatigue domain scores, whereas the pooled analysis showed no significant improvements in fatigue domain scores (WMD of $0.26,95 \%$ CI -0.11 to 


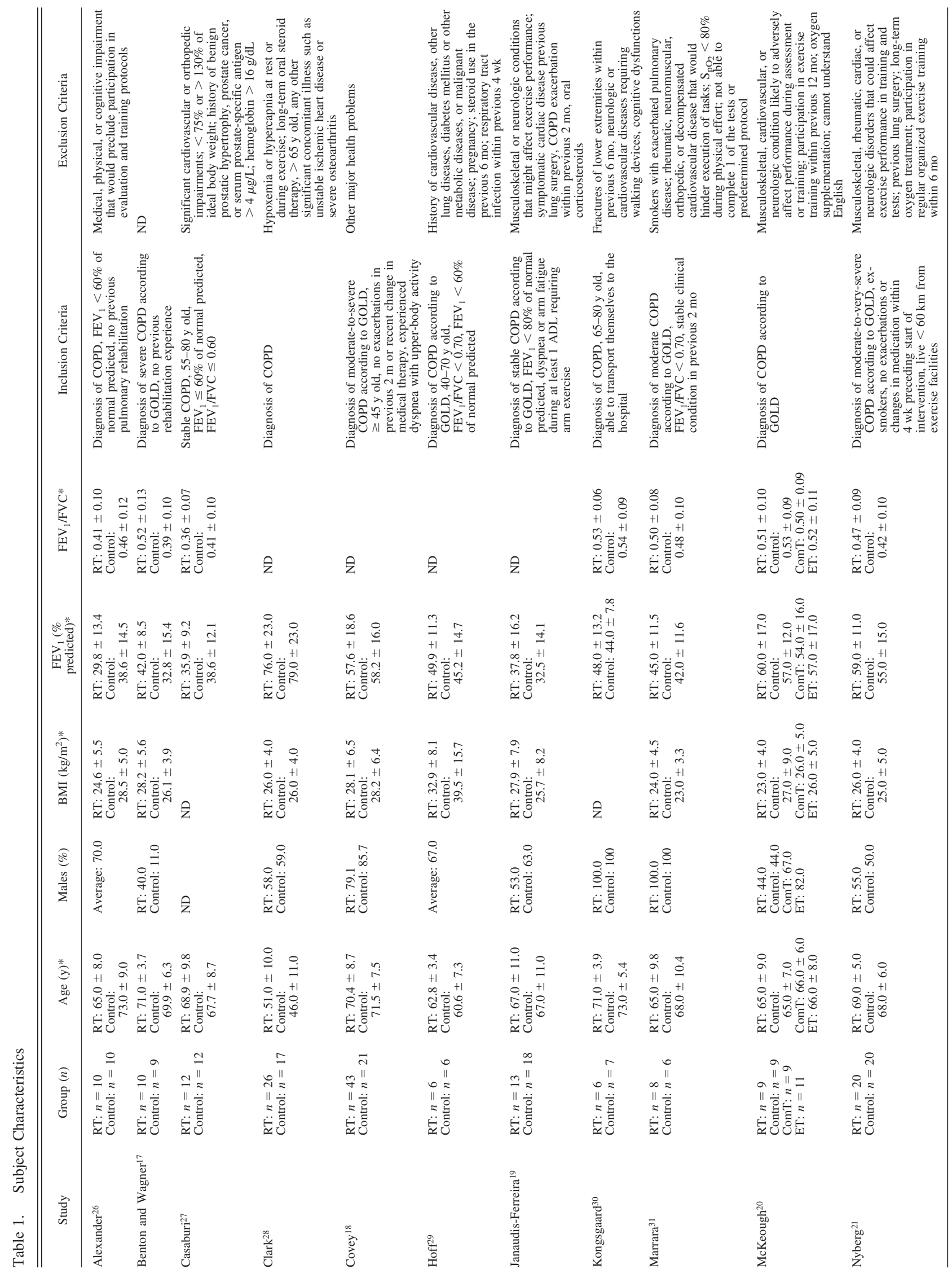


Resistance Training in Subjects With COPD

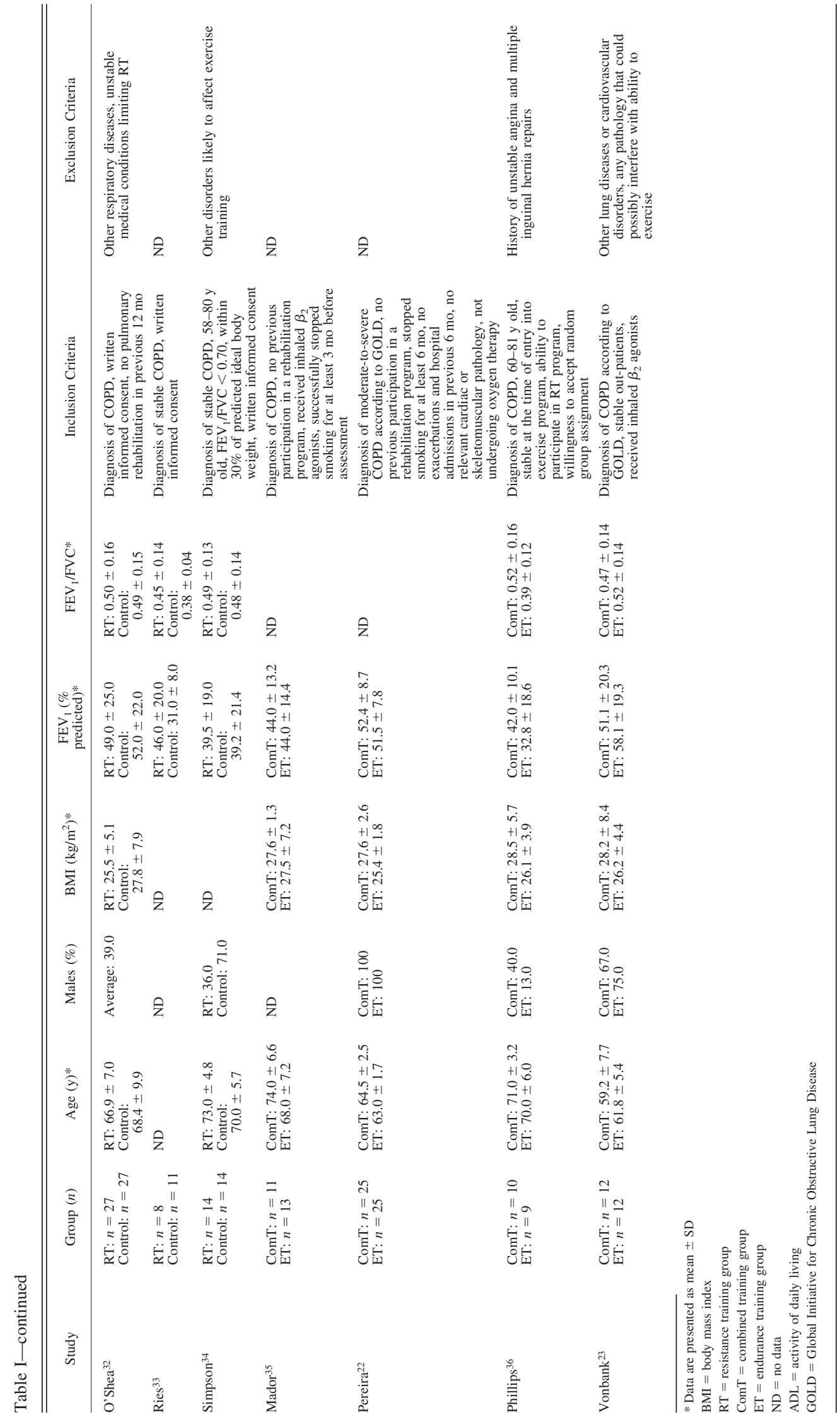




\section{Resistance Training in Subjects With COPD}

Table 2. Included Studies

\begin{tabular}{|c|c|c|c|c|c|}
\hline Study & Intervention & Study Design & Outcomes & $\begin{array}{c}\text { Jadad Scale } \\
\text { Score }\end{array}$ & $\begin{array}{l}\text { Attrition } \\
\text { Rate (\%) }\end{array}$ \\
\hline Alexander ${ }^{26}$ & $\begin{array}{l}\text { RT: } 5 \text { exercises, } 8-10 \mathrm{wk} \text {, twice/wk, } 1 \text { set/12 } \\
\text { reps, load of } 50 \% 1 \mathrm{RM} \text { (1st wk), increase } \\
\text { based on successful completion of }>12 \\
\text { reps for } 2 \text { consecutive training sessions in } \\
\text { 3-5-pound increments } \\
\text { Control: non-exercise } \\
\text { Baseline PR: identical intensity ET and low- } \\
\text { intensity upper-extremity RT for all subjects }\end{array}$ & RT vs control & $\begin{array}{l}\text { Exercise tolerance: } 6 \mathrm{MWD} \\
\text { Muscle strength: leg and incline bench } \\
\text { press } \\
\text { Other outcomes: functional fitness }\end{array}$ & 2 & 26 \\
\hline Benton and Wagner ${ }^{17}$ & $\begin{array}{l}\text { RT: } 5 \text { exercises, } 8 \text { wk, } 1 \text { set/8-12 reps, load of } \\
50 \% 1 \mathrm{RM} \text { for leg and chest press, with } \\
\text { other } 3 \text { exercises set at a weight that } \\
\text { allowed completion of } 10 \text { repetitions with } \\
\text { good form and without undue fatigue } \\
\text { Control: non-exercise } \\
\text { Baseline PR: identical intensity ET and low- } \\
\text { intensity upper-extremity RT for all subjects }\end{array}$ & RT vs control & $\begin{array}{l}\text { HRQOL: SF-36 } \\
\text { Exercise capacity: 6MWD } \\
\text { Muscle strength: incline chest and leg } \\
\text { press }\end{array}$ & 2 & 0 \\
\hline Casaburi ${ }^{27}$ & $\begin{array}{l}\text { RT: } 10 \mathrm{wk}, 3 \text { times/wk, } 3 \text { sets/12 reps } 1 \mathrm{st} \\
4 \mathrm{wk}, 4 \text { sets/8-10 reps last } 6 \mathrm{wk} \text {, loads of } \\
60 \% 1 \mathrm{RM}(1 \mathrm{st} 4 \mathrm{wk}) \text { and } 80 \% \text { 1RM (next } \\
6 \mathrm{wk}) \\
\text { Control: non-exercise }\end{array}$ & RT vs control & $\begin{array}{l}\text { Pulmonary function: } \mathrm{FEV}_{1}, \mathrm{FEV}_{1} \% \\
\text { predicted, } \mathrm{FEV}_{1} / \mathrm{FVC}\end{array}$ & 6 & 11 \\
\hline Clark $^{28}$ & $\begin{array}{l}\text { RT: } 8 \text { exercises, } 12 \text { wk, } 3 \text { sets } / 10 \text { reps, load of } \\
\quad 70 \% \text { of subject's maximum value } \\
\text { Control: non-exercise }\end{array}$ & RT vs control & $\begin{array}{l}\text { Physiologic parameters: } \dot{\mathrm{V}}_{\mathrm{O}_{2} *} \text {, heart } \\
\quad \text { rate, } \mathrm{V}_{\mathrm{E}}, \mathrm{V}_{\mathrm{T}} \text {, breathing frequency } \\
\text { Dyspnea: Borg dyspnea scale } \\
\text { Muscle strength: quadriceps }\end{array}$ & 2 & 0 \\
\hline Covey $^{18}$ & $\begin{array}{l}\text { RT: } 8 \text { exercises, } 16 \text { wk, twice/wk, } 2 \text { sets/8-10 } \\
\text { reps } 1 \text { st } 4 \mathrm{wk}, 3 \text { sets/8-10 reps next } \\
5-16 \text { wk, load of } 80 \% 1 \mathrm{RM} \\
\text { Control: sham training }\end{array}$ & RT vs control & $\begin{array}{l}\text { HRQOL: CRQ } \\
\text { Muscle strength: upper body } \\
\text { Other outcomes: } P_{\text {Imax }} \text {, functional } \\
\quad \text { status, self-efficacy }\end{array}$ & 7 & 19 \\
\hline $\operatorname{Hoff}^{29}$ & $\begin{array}{l}\text { RT: } 8 \text { wk, } 4 \text { sets } / 5 \text { reps, load of } 85-90 \% 1 \mathrm{RM} \text {, } \\
\text { increased by } 2.5 \mathrm{~kg} \text { until } 5 \text { repetitions could } \\
\text { again be achieved } \\
\text { Control: non-exercise }\end{array}$ & RT vs control & $\begin{array}{l}\text { Physiologic parameters: } \dot{\mathrm{V}}_{\mathrm{O}_{2}}, \dot{\mathrm{V}}_{\mathrm{E}} \text {, heart } \\
\text { rate, lactate, } \mathrm{S}_{\mathrm{aO}_{2}} \text {, maximum work } \\
\text { capacity } \\
\text { Muscle strength: quadriceps } \\
\text { Other outcomes: RPE }\end{array}$ & 2 & 0 \\
\hline Janaudis-Ferreira $^{19}$ & $\begin{array}{l}\text { RT: } 6 \mathrm{wk}, 3 \text { times/wk, } 1 \text { set/10-12 reps, load } \\
\text { of loads equivalent to the } 10-12 \text {-rep } \\
\text { maximum (if completed, loads were } \\
\text { increased) } \\
\text { Control: sham training } \\
\text { Baseline PR: identical intensity ET, RT, and } \\
\text { breathing exercises for all subjects }\end{array}$ & Arm RT vs control & $\begin{array}{l}\text { HRQOL: CRQ } \\
\text { Dyspnea: dyspnea domain of CRQ } \\
\text { Muscle strength: elbow flexion and } \\
\text { extension, shoulder flexion and } \\
\text { abduction } \\
\text { Other outcomes: arm function, arm } \\
\text { exercise capacity }{ }^{16}\end{array}$ & 6 & 6 \\
\hline Kongsgaard ${ }^{30}$ & $\begin{array}{l}\text { RT: } 12 \mathrm{wk} \text {, twice/wk, } 4 \text { sets } / 8 \text { reps, load of } \\
80 \% 1 \mathrm{RM} \\
\text { Control: non-exercise }\end{array}$ & RT vs control & $\begin{array}{l}\text { Pulmonary function: } \mathrm{FEV}_{1} \\
\text { Muscle strength: knee extension, } \\
\text { trunk, leg extension power } \\
\text { Other outcomes: CSA of quadriceps, } \\
\text { normal and maximum gait speed, } \\
\text { stair-climbing time }\end{array}$ & 2 & 28 \\
\hline Marrara $^{31}$ & $\begin{array}{l}\text { RT: } 6 \text { exercises, } 6 \text { wk, } 3 \text { times/wk, } 3 \text { sets } / 10 \\
\text { reps, load of } 50 \% \text { of } 10 \mathrm{RM} \text { (1st set), load } \\
\text { of } 75 \% \text { of } 10 \mathrm{RM} \text { ( } 2 \mathrm{nd} \text { set), } 100 \% \text { of load } \\
\text { of } 10 \mathrm{RM} \text { (3rd set) } \\
\text { Control: non-exercise }\end{array}$ & RT vs control & $\begin{array}{l}\text { Physiologic parameters: } \dot{\mathrm{V}}_{\mathrm{E}} / \mathrm{MVV}(\%), \\
\dot{\mathrm{V}}_{\mathrm{O}_{2}} / \text { maximum } \dot{\mathrm{V}}_{\mathrm{O}_{2}}(\%) \text { during } \\
\text { daily physical activities test } \\
\text { Dyspnea: Borg dyspnea scale during } \\
\text { daily physical activities test }\end{array}$ & 1 & 24 \\
\hline McKeough $^{20}$ & $\begin{array}{l}\text { Arm RT: } 8 \text { wk, } 3 \text { times/wk, } 2 \text { sets } / 10 \text { reps to } 3 \\
\text { sets/10 reps, load of } 60 \% \text { 1RM to } 80 \% \\
\text { 1RM } \\
\text { Arm ET: arm cranking and unsupported arm } \\
\text { exercise, } 8 \text { wk, } 3 \text { times/wk, } 60 \% \text { work rate } \\
\text { of peak arm crank test for } 15 \text { min/session } \\
\text { and } 1 \text { level below the maximum level } \\
\text { achieved on the unsupported arm test for } \\
5 \text { min/session, intensity increased according } \\
\text { to breathlessness and perceived arm } \\
\text { exertion } \\
\text { Arm ComT: arm RT plus arm ET } \\
\text { Arm control: non-exercise } \\
\text { Baseline PR: identical intensity RT and ET of } \\
\text { lower extremities for all subjects }\end{array}$ & $\begin{array}{l}\text { Arm RT vs control } \\
\text { Arm ComT vs arm } \\
\text { ET }\end{array}$ & $\begin{array}{l}\text { Physiologic parameters: } \dot{\mathrm{V}}_{\mathrm{O}_{2}}, \dot{\mathrm{V}}_{\mathrm{E}} \text {, } \\
\dot{\mathrm{V}}_{\mathrm{CO}_{2}} \\
\text { HRQOL: SGRQ } \\
\text { Dyspnea: Borg scores } \\
\text { Other outcomes: Functional arm } \\
\quad \text { exercise testing }\end{array}$ & 6 & 27 \\
\hline Nyberg 21 & $\begin{array}{l}\text { RT: } 8 \text { exercises, } 8 \mathrm{wk}, 3 \text { times/wk, } 2 \text { sets } / 25 \\
\text { reps, load individually determined and } \\
\text { progressed using Borg category ratio scale } \\
\text { Control: non-exercise }\end{array}$ & RT vs control & $\begin{array}{l}\text { Physiologic parameters: } \dot{\mathrm{V}}_{\mathrm{O}_{2}} \\
\text { HRQOL: CRQ, SF-36 } \\
\text { Exercise capacity: 6MWD, 6PBRT } \\
\text { Muscle strength: knee extensor, } \\
\text { shoulder flexion }\end{array}$ & 4 & 9 \\
\hline O'Shea ${ }^{32}$ & $\begin{array}{l}\text { RT: } 6 \text { exercises, } 12 \mathrm{wk}, 3 \text { times/wk, } 3 \text { sets } / 8- \\
12 \text { reps, load of maximum to complete } \\
\text { sets/reps } \\
\text { Control: non-exercise }\end{array}$ & RT vs control & $\begin{array}{l}\text { HRQOL: CRQ } \\
\text { Exercise capacity: 6MWD } \\
\text { Muscle strength: knee extensor, hip } \\
\text { abductor, shoulder horizontal flexor, } \\
\text { shoulder flexor } \\
\text { Other outcomes: mobility, upper-limb } \\
\text { activity, participation restrictions }\end{array}$ & 3 & 19 \\
\hline
\end{tabular}




\section{Resistance Training in Subjects With COPD}

Table 2. Included Studies

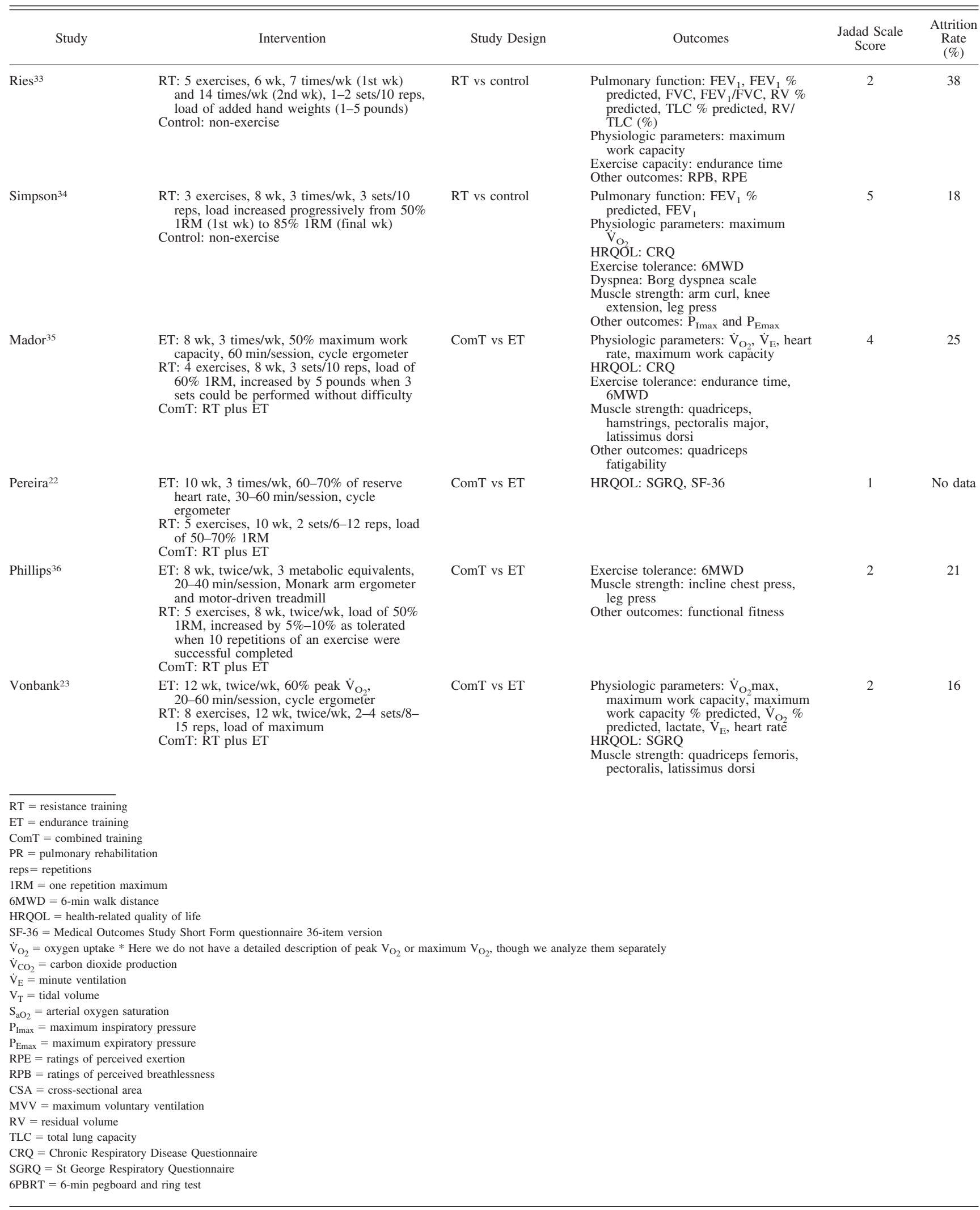


Resistance Training in Subjects With COPD

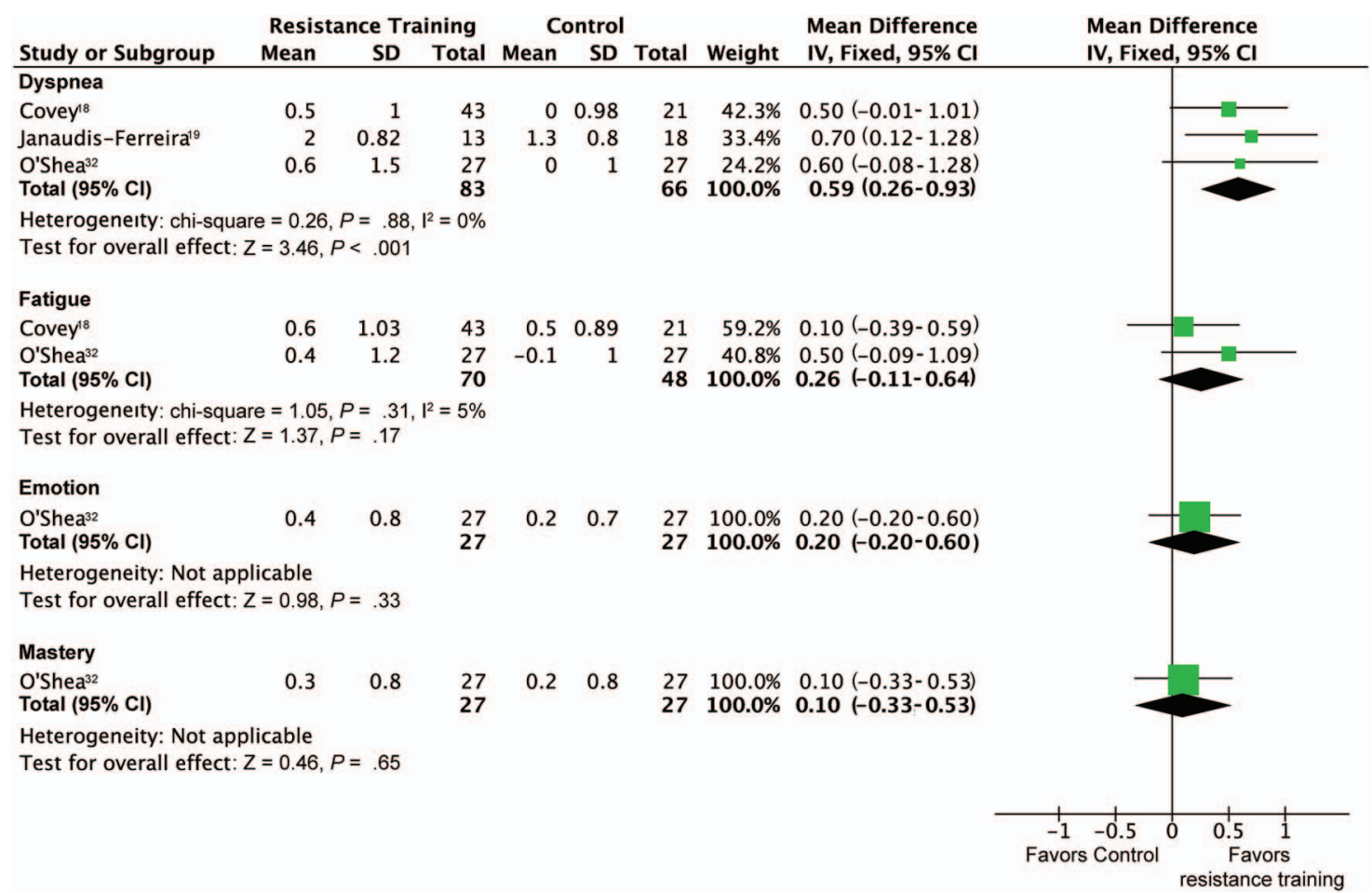

Fig. 2. Effects of resistance training vs non-exercise control on Chronic Respiratory Disease Questionnaire scores. IV = inverse variance weighting.

$\left.0.64, \mathrm{I}^{2}=5 \%, P=.17\right) .{ }^{18,32}$ Only one study reported CRQ emotion and mastery domain scores. ${ }^{32}$ The results showed no significant difference in CRQ emotion domain scores $(\mathrm{d}=0.20,95 \% \mathrm{CI}-0.20$ to 0.60$)$ and mastery domain scores $(\mathrm{d}=0.10,95 \% \mathrm{CI}-0.33$ to 0.53$)$ between the 2 groups (Fig. 2).

Resistance-and-Endurance-Training Group Versus Endurance-Training-Alone Group. Only one trial reported each CRQ domain score. ${ }^{35}$ There were no significant differences in dyspnea domain scores $(\mathrm{d}=-0.60$, $95 \%$ CI -1.23 to 0.03$)$, fatigue domain scores $(\mathrm{d}=$ $-0.30,95 \%$ CI -1.18 to 0.58 ), emotion domain scores $(\mathrm{d}=0.00,95 \% \mathrm{CI}-0.74$ to 0.74$)$, and mastery domain scores $(\mathrm{d}=0.10,95 \% \mathrm{CI}-0.89$ to 1.09$)$ between the 2 groups.

\section{St George Respiratory Questionnaire}

Resistance-Training Group Versus Non-Exercise Control Group. Only one included trial reported St George Respiratory Questionnaire (SGRQ) total scores and each domain score. ${ }^{20}$ The results showed no statistically significant improvements in SGRQ total scores $(\mathrm{d}=-3$, $95 \% \mathrm{CI}-14$ to 8$)$, symptom domain scores $(\mathrm{d}=-7$, $95 \%$ CI -23 to 9), activity domain scores ( $d=$ $-0.1,95 \% \mathrm{CI}-15$ to 15$)$, and impact domain scores $(\mathrm{d}=-3,95 \% \mathrm{CI}-16$ to 10$)$ in the resistance-training group.
Resistance-and-Endurance-Training Group Versus Endurance-Training-Alone Group. Three studies reported SGRQ total scores, ${ }^{20,22,23}$ and 2 studies reported each SGRQ domain score. ${ }^{22,23}$ The results of 2 included studies showed significant improvements in SGRQ total scores, symptom domain scores, activity domain scores, and impact domain scores in the resistance-and-endurancetraining group. The overall analysis showed statistically significant improvements in SGRQ total scores (WMD of $-7.44,95 \%$ CI -12.62 to $\left.-2.25, \mathrm{I}^{2}=0 \%, P=.005\right)$, symptom domain scores (WMD of $-14.81,95 \%$ CI -21.23 to $\left.-8.39, \mathrm{I}^{2}=0 \%, P<.001\right)$, activity domain scores (WMD of $-25.27,95 \%$ CI -31.46 to $-19.08, \mathrm{I}^{2}=11 \%$, $P<.001$ ), and impact domain scores (WMD of -8.23 , $95 \% \mathrm{CI}-15.31$ to $-1.15, \mathrm{I}^{2}=0 \%, P=.02$ ), favoring the combination training (Fig. 3).

\section{Secondary Outcomes}

\section{Skeletal Muscle Function}

Resistance-Training Group Versus Non-Exercise Control Group. The cumulative analysis showed significant improvements in knee extension strength (WMD of $7.78 \mathrm{~kg}, \quad 95 \% \quad$ CI $5.18-10.38 \mathrm{~kg}, \quad \mathrm{I}^{2}=0 \%$, $P<.001$ ), ${ }^{28,32,34}$ leg press strength (WMD of $16.67 \mathrm{~kg}$, $95 \%$ CI $\left.2.87-30.47 \mathrm{~kg}, \mathrm{I}^{2}=0 \%, P=.02\right),{ }^{17,26,27,29,34}$ and shoulder flexion strength (WMD of $2.88 \mathrm{~kg}$, $95 \%$ CI $\left.0.56-5.20 \mathrm{~kg}, \mathrm{I}^{2}=0 \%, P=.01\right)^{19,32}$ in the 


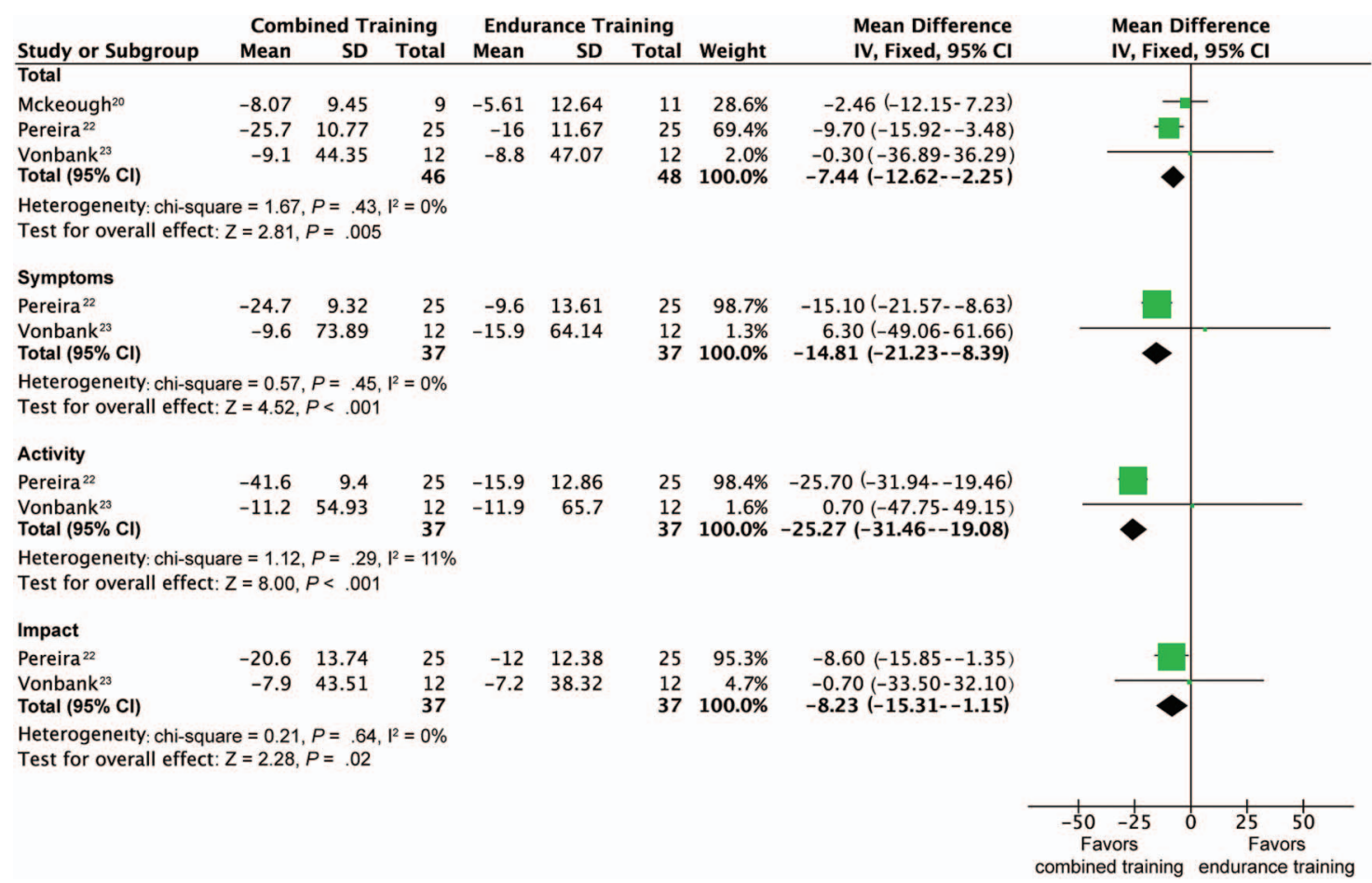

Fig. 3. Effects of combined resistance and endurance training vs endurance training alone on St George Respiratory Questionnaire scores. IV = inverse variance weighting.

resistance-training groups. However, the difference was not statistically significant in pectoral muscle strength (WMD of $2.29 \mathrm{~kg}, 95 \% \mathrm{CI}-0.41$ to $4.99 \mathrm{~kg}, \mathrm{I}^{2}=0 \%$, $P=.10$ ) after resistance training (Fig. 4). ${ }^{17,26,32}$ Only one study measured latissimus dorsi strength, which showed a significant improvement $(\mathrm{d}=2.50 \mathrm{~kg}$, $95 \% \mathrm{CI}-0.70$ to $5.70 \mathrm{~kg}$ ) in the resistance-training group. ${ }^{19}$

Resistance-and-Endurance-Training Group Versus Endurance-Training-Alone Group. The cumulative analysis showed significant improvements in leg press strength (WMD of $12.34 \mathrm{~kg}, 95 \%$ CI $5.96-18.72 \mathrm{~kg}$, $\left.\mathrm{I}^{2}=0 \%, P<.001\right)^{23,36}$ and pectoral muscle strength (WMD of $4.48 \mathrm{~kg}, 95 \%$ CI $2.53-6.43 \mathrm{~kg}, \mathrm{I}^{2}=0 \%$, $P<.001)^{23,35,36}$ in the resistance-and-endurance-training group compared with the endurance-training-along group. No significant difference in latissimus dorsi strength (WMD of $6.07 \mathrm{~kg}, 95 \% \mathrm{CI}-3.22$ to $15.37 \mathrm{~kg}, \mathrm{I}^{2}=0 \%, P=.20$ ) was observed after the addition of resistance training to endurance training (Fig. 5).23,35 Only one study measured knee extension strength $(\mathrm{d}=10.00 \mathrm{~kg}, 95 \% \mathrm{CI}-1.53$ to $21.53 \mathrm{~kg}, P<.002) .35$

\section{6-min Walk Distance}

Resistance-Training Group Versus Non-Exercise Control Group. Five studies included the 6-min walk distance (6MWD) as an end point. ${ }^{17,21,26,32,34}$ The results of each study and of our pooled analysis showed no significant difference in 6MWD (WMD of $1.83 \mathrm{~m}, 95 \% \mathrm{CI}-15.32$ to $18.97 \mathrm{~m}$, $\mathrm{I}^{2}=0 \%, P=.83$ ) between the 2 groups.

Resistance-and-Endurance-Training Group Versus Endurance-Training-Alone Group. Two included trials reported 6MWD. ${ }^{35,36}$ The results of each study showed no significant improvements in 6MWD in the resistanceand-endurance-training group. The pooled analysis showed no obvious changes in 6MWD between the 2 groups (WMD of $-1.94 \mathrm{~m}, 95 \% \mathrm{CI}-49.55$ to $45.67 \mathrm{~m}, \mathrm{I}^{2}=0 \%$, $P=.94)$.

\section{6-min Pegboard and Ring Test}

Resistance-Training Group Versus Non-Exercise Control Group. Two included trials ${ }^{19,21}$ reported results from the 6-min pegboard and ring test. The cumulative analysis showed no significant difference between the 2 groups (WMD of 20.52 rings, $95 \%$ CI -2.54 to 43.58 rings, $\mathrm{I}^{2}=0 \%, P=.08$ ).

\section{Maximum Exercise Work Load}

Resistance-Training Group Versus Non-Exercise Control Group. Two included trials reported the maximum exercise work load. ${ }^{27,29}$ The results of each study 
Resistance Training in Subjects With COPD

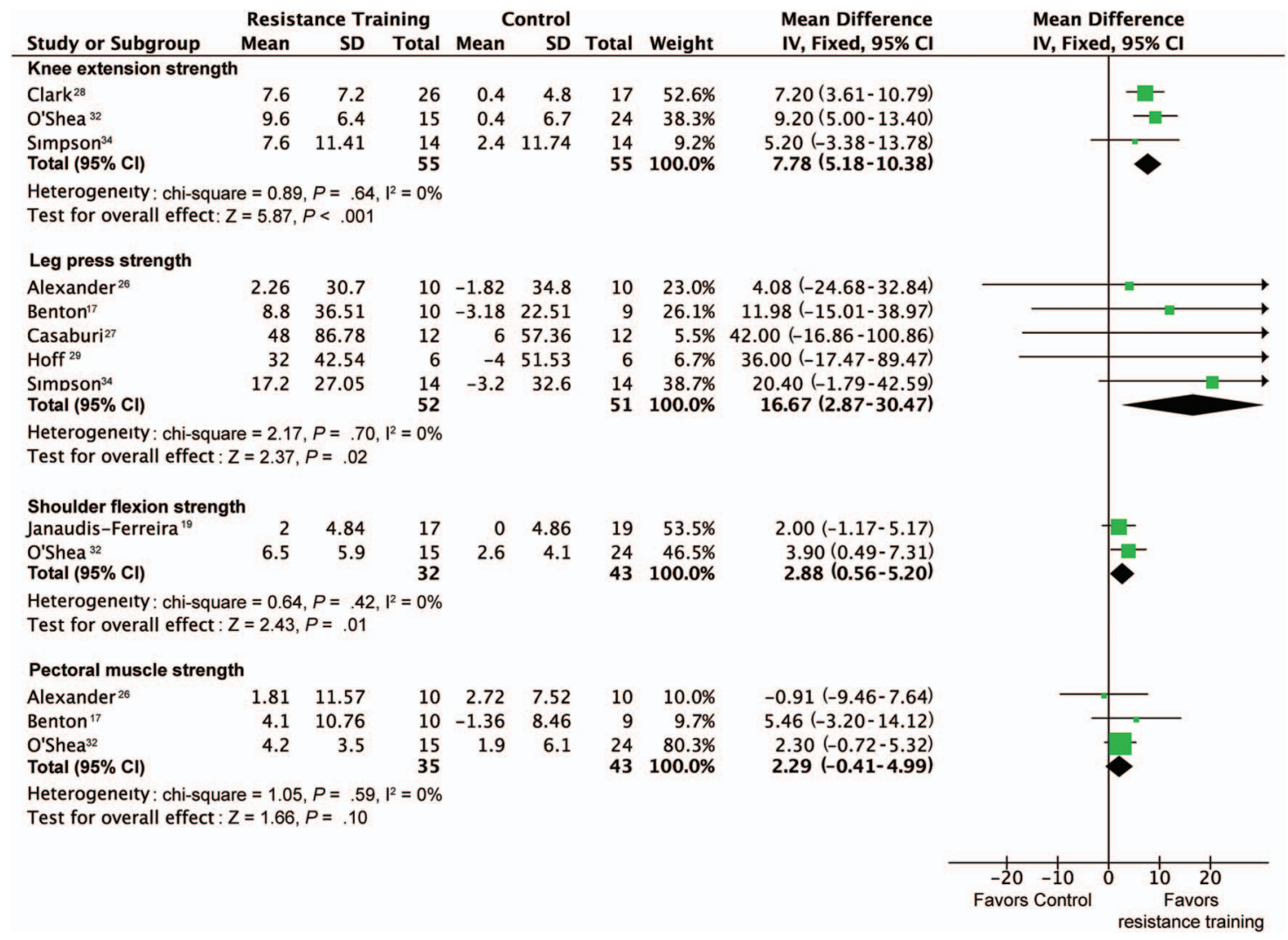

Fig. 4. Effects of resistance training vs non-exercise control on skeletal muscle strength. IV = inverse variance weighting.

\begin{tabular}{|c|c|c|c|c|c|c|c|c|c|c|}
\hline \multirow{2}{*}{$\begin{array}{l}\text { Study or Subgroup } \\
\text { Leg press strength }\end{array}$} & \multicolumn{3}{|c|}{ Combined Training } & \multicolumn{3}{|c|}{ Endurance Training } & Weight & $\begin{array}{l}\text { Mean Difference } \\
\text { IV, Fixed, } 95 \% \mathrm{CI}\end{array}$ & \multicolumn{2}{|c|}{$\begin{array}{l}\text { Mean Difference } \\
\text { IV, Fixed, } 95 \% \mathrm{Cl}\end{array}$} \\
\hline & & & & & & & & & & \\
\hline Philips ${ }^{36}$ & 9.07 & 8.6 & 10 & -3.18 & 5.43 & 9 & $99.3 \%$ & $12.25(5.85-18.65)$ & & \\
\hline $\begin{array}{l}\text { Vonbank } \\
\text { Total }(95 \% \mathrm{Cl})\end{array}$ & 38.3 & 111.68 & $\begin{array}{l}12 \\
22\end{array}$ & 12.8 & 81.66 & $\begin{array}{l}12 \\
21\end{array}$ & $\begin{array}{r}0.7 \% \\
100.0 \%\end{array}$ & $\begin{array}{l}25.50(-52.78-103.78) \\
12.34(5.96-18.72)\end{array}$ & & \\
\hline \multicolumn{11}{|c|}{$\begin{array}{l}\text { Heterogeneity: chi-square }=0.11, P=.74, I^{2}=0 \% \\
\text { Test for overall effect: } Z=3.79, P<.001\end{array}$} \\
\hline \multicolumn{11}{|c|}{ Pectoral muscle strength } \\
\hline Mador ${ }^{35}$ & 5 & 13.67 & 11 & 2 & 11.74 & 13 & $3.6 \%$ & $3.00(-7.29-13.29)$ & & 一 \\
\hline Philips ${ }^{36}$ & 4.08 & 1.42 & 10 & -0.45 & 2.73 & 9 & $96.2 \%$ & $4.53(2.54-6.52)$ & & \\
\hline $\begin{array}{l}\text { Vonbank } \\
\text { Total }(95 \% \mathrm{Cl})\end{array}$ & 9 & 54.28 & 12 & 2.3 & 40.29 & $\begin{array}{l}12 \\
34\end{array}$ & $\begin{array}{r}0.3 \% \\
100.0 \%\end{array}$ & $\begin{array}{r}6.70(-31.55-44.95) \\
4.48(2.53-6.43)\end{array}$ & & 1 \\
\hline \multicolumn{11}{|c|}{$\begin{array}{l}\text { Heterogeneity: chi-square }=0.09, P=.95, I^{2}=0 \% \\
\text { Test for overall effect: } Z=4.50, P<.001\end{array}$} \\
\hline \multicolumn{11}{|c|}{ Latissimus dorsi strength } \\
\hline Mador ${ }^{35}$ & 5 & 11.87 & 11 & -1 & 11.74 & 13 & $96.1 \%$ & $6.00(-3.48-15.48)$ & & 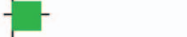 \\
\hline $\begin{array}{l}\text { Vonbank }{ }^{23} \\
\text { Total }(95 \% \mathrm{Cl})\end{array}$ & 11.6 & 67.51 & 12 & 3.8 & 47.72 & $\frac{12}{25}$ & $\begin{array}{r}3.9 \% \\
100.0 \%\end{array}$ & $\begin{array}{r}7.80(-38.98-54.58) \\
6.07(-3.22-15.37)\end{array}$ & & \\
\hline \multicolumn{11}{|c|}{$\begin{array}{l}\text { Heterogeneity: chi-square }=0.01, P=.94, I^{2}=0 \% \\
\text { Test for overall effect: } Z=1.28, P=.20\end{array}$} \\
\hline & & & & & & & & & $\begin{array}{l}-50-25 \\
\text { Favors } \\
\text { durance training }\end{array}$ & $\begin{array}{ccc}0 & 25 & 50 \\
& & 1 \\
& \text { Favors } \\
\text { g combined training }\end{array}$ \\
\hline
\end{tabular}

Fig. 5. Effects of combined resistance and endurance training vs endurance training alone on skeletal muscle strength. IV $=$ inverse variance weighting.

showed no significant difference, and the overall analysis also showed no significant difference between the 2 groups (WMD of $3.46 \mathrm{~W}, 95 \% \mathrm{CI}-16.75$ to $23.67 \mathrm{~W}$, $\left.\mathrm{I}^{2}=0 \%, P=.74\right)$.
Resistance-and-Endurance-Training Group Versus Endurance-Training-Alone Group. Two included trials reported the maximum exercise work load. ${ }^{23,35}$ The results of each study showed no significant difference, and 


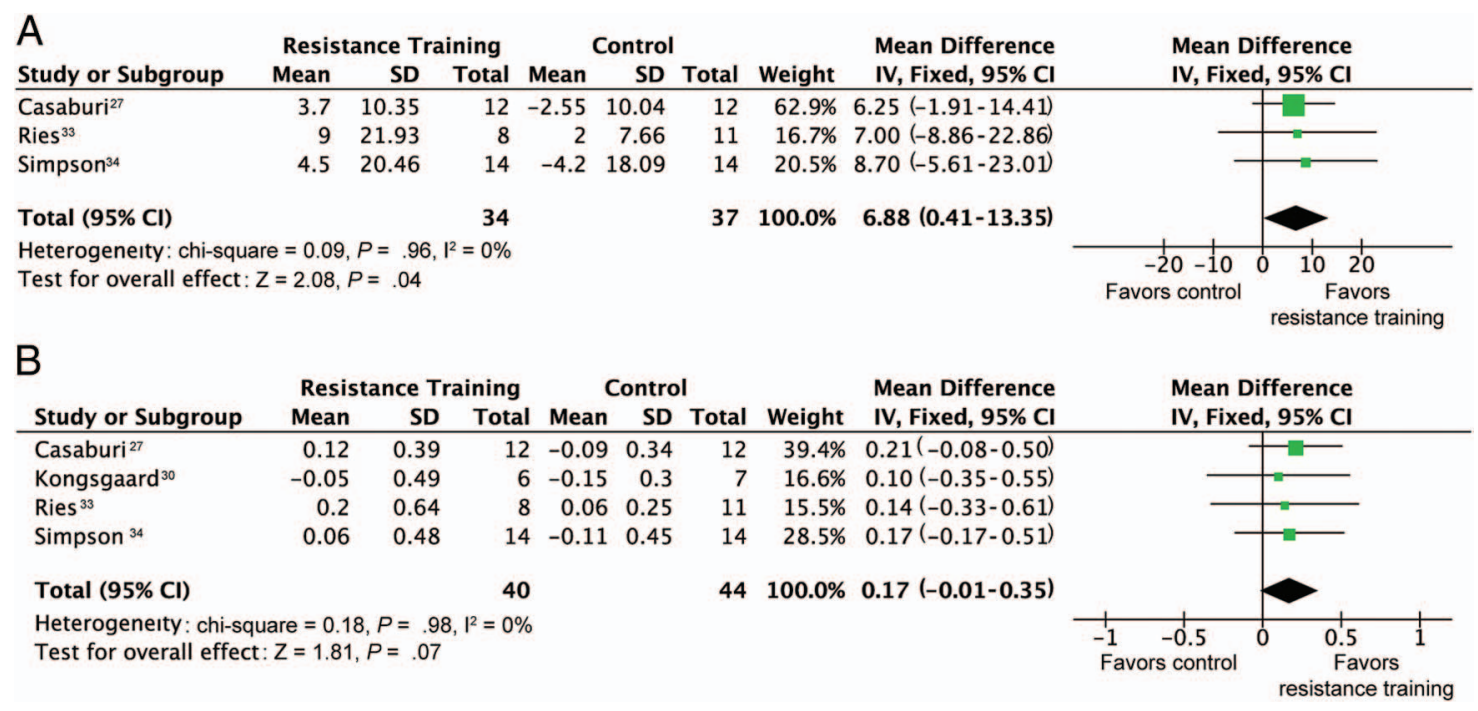

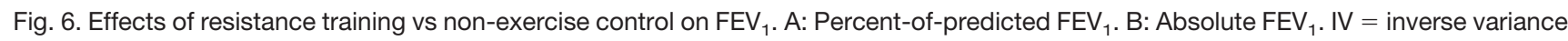
weighting.

the overall analysis also showed no significant difference between the 2 groups (WMD of $2.91 \mathrm{~W}, 95 \% \mathrm{CI}-18.03$ to $23.85 \mathrm{~W}, \mathrm{I}^{2}=0 \%, P=.79$ ).

\section{Maximum Oxygen Consumption}

Resistance-Training Group Versus Non-Exercise Control Group. Three included trials reported maximum oxygen consumption. ${ }^{20,27,29}$ The results of each study showed no significant difference, and the pooled analysis also showed no significant difference between the 2 groups (WMD of $0.04 \mathrm{~L} / \mathrm{min}, 95 \% \mathrm{CI}-0.13$ to $0.21 \mathrm{~L} / \mathrm{min}$, $\left.\mathrm{I}^{2}=0 \%, P=.61\right)$.

Resistance-and-Endurance-Training Group Versus Endurance-Training-Alone Group. Two studies reported maximum oxygen consumption. ${ }^{20,35}$ The results of each study showed no significant difference, and the pooled analysis also showed no significant difference between the 2 groups (WMD of $0.02 \mathrm{~L} / \mathrm{min}, 95 \% \mathrm{CI}-0.16$ to $0.21 \mathrm{~L} /$ $\left.\min , \mathrm{I}^{2}=0 \%, P=.79\right)$.

\section{Pulmonary Function}

\section{Change in $\mathrm{FEV}_{1}$}

Resistance-Training Group Versus Non-Exercise Control Group. Three trials reported percent-ofpredicted $\mathrm{FEV}_{1}, 27,33,34$ and 4 trials reported absolute $\mathrm{FEV}_{1} \cdot{ }^{27,30,33,34}$ The pooled analysis showed significant improvements in percent-of-predicted $\mathrm{FEV}_{1}$ (WMD of $6.88 \%$, $95 \%$ CI $0.41-13.35 \%, \mathrm{I}^{2}=0 \%, P=.04$ ) in the resistancetraining groups compared with the non-exercise control groups. For absolute $\mathrm{FEV}_{1}$, the difference between the 2 groups was not statistically significant (WMD of $0.17 \mathrm{~L}$, 95\% CI -0.01 to $0.35 \mathrm{~L}, \mathrm{I}^{2}=0 \%, P=.07$ ) (Fig. 6).

Only one trial reported absolute FVC. ${ }^{33}$ The results showed significant improvements in the resistance-training group $(\mathrm{d}=0.11 \mathrm{~L}, 95 \% \mathrm{CI}-0.62$ to $0.84 \mathrm{~L}, P<.05)$.

\section{Attrition Rate and Adverse Events}

The attrition rate was reported in 17 included studies. The mean attrition rate was $16.9 \%$, ranging from 0 to $38.0 \%$. The main reasons for withdrawal included COPD exacerbations (17/119), failure to complete the program (21/119), non-protocol-related or non-COPD-related health problems (34/119), personal reasons (34/119), refusal of post-rehabilitation measurements (5/119), musculoskeletal problems (4/119), treatment changes (3/119), and generalized weakness (1/119). The pooled analysis showed that the attrition rate was higher in the resistance-training group compared with the non-exercise control group (odds ratio of $1.79,95 \%$ CI $1.04-3.08, \mathrm{I}^{2}=0 \%, P=.03$ ) (Fig. 7). No significant difference in the attrition rate between the resistance-and-endurance-training and endurance-trainingalone groups (odds ratio of $1.15,95 \%$ CI $0.32-4.15$, $\mathrm{I}^{2}=0 \%, P=.83$ ) was observed (Fig. 8). No significant changes were observed in reasons for withdrawal between the resistance-training and non-exercise control groups (Fig. 9). There were no reports of adverse events related to resistance-training intervention. The overall analysis showed no obvious difference in reasons for withdrawal between the resistance-and-endurance-training and endurance-training-alone groups (Fig. 10). 


\section{Resistance Training in Subjects With COPD}

\begin{tabular}{|c|c|c|c|c|c|c|c|c|}
\hline Study or Subgroup & \multicolumn{2}{|c|}{ Resistance Training } & \multicolumn{2}{|c|}{ Control } & Weight & $\begin{array}{c}\text { Odds Ratio } \\
\text { M-H, Fixed, } 95 \% \mathrm{Cl}\end{array}$ & \multicolumn{2}{|c|}{$\begin{array}{c}\text { Odds Ratio } \\
\text { M-H, Fixed, 95\% Cl }\end{array}$} \\
\hline Alexander ${ }^{26}$ & 5 & 15 & 2 & 12 & $7.3 \%$ & $2.50(0.39-16.05)$ & & \\
\hline Casaburi ${ }^{27}$ & 1 & 13 & 1 & 13 & $4.6 \%$ & $1.00(0.06-17.90)$ & & \\
\hline Covey ${ }^{18}$ & 14 & 57 & 7 & 28 & $35.1 \%$ & $0.98(0.34-2.78)$ & & \\
\hline Janaudis-Ferreira $^{19}$ & 4 & 17 & 1 & 19 & $3.6 \%$ & $5.54(0.55-55.49)$ & & \\
\hline Kongsgaard ${ }^{30}$ & 3 & 9 & 2 & 9 & $6.6 \%$ & $1.75(0.22-14.22)$ & & \\
\hline Mckeough 20 & 5 & 14 & 4 & 13 & $13.2 \%$ & $1.25(0.25-6.23)$ & & \\
\hline Nyberg 21 & 2 & 22 & 2 & 22 & $9.0 \%$ & $1.00(0.13-7.81)$ & & \\
\hline O'Shea ${ }^{32}$ & 12 & 27 & 3 & 27 & $8.3 \%$ & $6.40(1.55-26.48)$ & & \\
\hline Simpson ${ }^{34}$ & 3 & 17 & 3 & 17 & $12.3 \%$ & $1.00(0.17-5.83)$ & & \\
\hline Total (95\% Cl) & & 191 & & 160 & $100.0 \%$ & $1.79(1.04-3.08)$ & & \\
\hline Total events & 49 & & 25 & & & & & \\
\hline $\begin{array}{l}\text { Heterogeneity: chi-so } \\
\text { Test for overall effec }\end{array}$ & $\begin{array}{l}=6.50, P= \\
=2.12, P=\end{array}$ & $9,1^{2}=0 \%$ & & & & & $\begin{array}{l}0.02 \quad 0.1 \\
\quad \text { Favors } \\
\text { resistance training }\end{array}$ & $\begin{array}{cr}10 & 50 \\
\text { Favors control }\end{array}$ \\
\hline
\end{tabular}

Fig. 7. Effects of resistance training vs non-exercise control on attrition rates. $\mathrm{M}-\mathrm{H}=$ Mantal-Haenszel statistics.

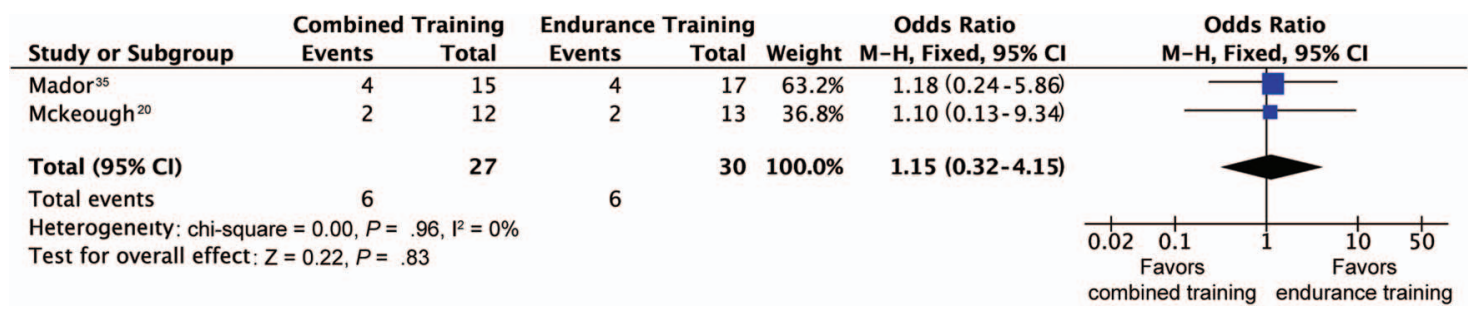

Fig. 8. Effects of combined resistance and endurance training vs endurance training alone on attrition rates. $\mathrm{M}-\mathrm{H}=\mathrm{Mantal}-\mathrm{Haenszel}$ statistics.

\section{Discussion}

Exercise training provides an effective therapy for exercise limitation in patients with COPD. The conventional modalities of exercise training include mainly endurance and resistance training. Endurance training is recommended by various guidelines as the cornerstone of successful pulmonary rehabilitation. ${ }^{38-40}$ Although increases in muscle strength after resistance training were demonstrated in subjects with COPD, ${ }^{41}$ the effect of resistance training and combined resistance and endurance training on clinically relevant outcomes in patients with COPD remains controversial. This meta-analysis incorporated 18 RCTs and included data from 750 subjects with advanced COPD. The effects of resistance training and combined resistance and endurance training were evaluated by their impact on quality of life, dyspnea, functional exercise capacity, maximum exercise capacity, skeletal muscle function, lung function, and adverse events. To our knowledge, this is the largest analysis to date of the efficacy of resistance training on clinically relevant outcomes in subjects with COPD.

This meta-analysis clearly showed the beneficial effects of resistance training on skeletal muscle strength in subjects with COPD. The results support previous findings. ${ }^{13,14,16} \mathrm{O}$ 'Shea et $\mathrm{al}^{14}$ reported that there were obvious increases in knee extension strength, leg press strength, and latissimus dorsi strength following resistance training versus no exercise (control). We did not perform cumulative analysis of latissimus dorsi strength because there was only one suitable study. O'Shea et al ${ }^{14}$ included some non-RCTs. In addition, they included all studies with resistance training, including resistance training versus nonexercise control, resistance training versus endurance training, resistance training versus resistance plus endurance training, resistance plus endurance training versus nonexercise control, and resistance plus endurance training versus endurance training. Thus, there may be a higher risk of heterogeneity in their analysis. Actually, O'Shea et $\mathrm{al}^{14}$ did not report the effect of resistance training on pectoral muscle strength because statistical heterogeneity between trials prevented the use of meta-analysis. More importantly, they reported the percentage increase in skeletal muscle strength, and we reported the absolute value of skeletal muscle strength. Other important findings of our study include the beneficial effects of resistance training on shoulder flexion strength and CRQ dyspnea domain scores. The improvement in CRQ dyspnea domain scores achieved a minimum clinically important difference of 0.5 units. Despite the positive effects of resistance training on skeletal muscle strength and CRQ dyspnea domain scores, there were no significant differences between the 2 groups in functional exercise capacity (including 6MWD and 6-min pegboard and ring test scores) and maximum exercise capacity (including maximum exercise work load 


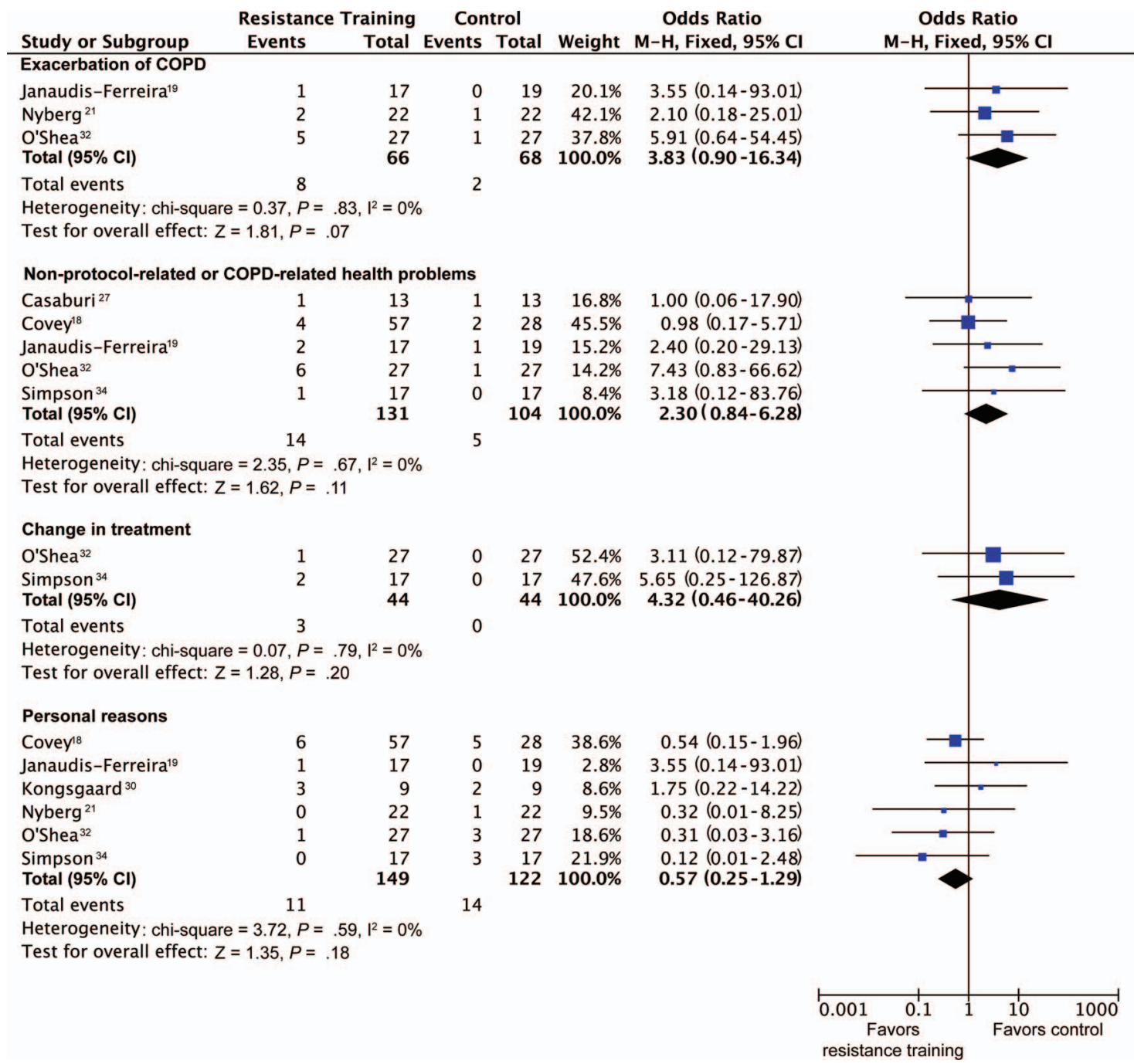

Fig. 9. Effects of resistance training vs non-exercise control on reasons for withdrawal. $\mathrm{M}-\mathrm{H}=\mathrm{Mantal}-\mathrm{Haenszel}$ statistics.

and maximum oxygen consumption). Our results support resistance training performed in conjunction with endurance training because the combination may improve skeletal muscle strength and quality of life to a greater degree than endurance training alone in patients with COPD. However, gains in skeletal muscle strength and quality of life failed to translate into improvements in exercise capacity. The mechanisms of intrinsic muscle changes after resistance training have been scarcely studied in COPD. ${ }^{41}$ Some authors speculated that the changes were related to the expression of muscle insulin-like growth factor-1 and myogenic regulatory factors. ${ }^{42}$ Additional studies are required to examine the mechanisms of intrinsic muscle changes after resistance training, which should greatly improve the clinical outcomes in patients with COPD.

It has been generally accepted that pulmonary rehabilitation by itself does not improve lung function. ${ }^{43,44} \mathrm{~A}$ meta-analysis conducted by Strasser et al ${ }^{15}$ showed that resistance training did not increase $\mathrm{FEV}_{1}$ but may carry potential benefits for FVC. Because of the inclusion of all studies with resistance training, there was a high heterogeneity with regard to percent-of-predicted $\mathrm{FEV}_{1}$ $\left(\mathrm{I}^{2}=68.1 \%\right)$. Our results showed that there was an obvious improvement in percent-of-predicted $\mathrm{FEV}_{1}$ and an increasing trend of absolute $\mathrm{FEV}_{1}$ in the resistance-training group. Although this phenomenon had been reported previously, ${ }^{15,45,46}$ we did not consider it to be a direct consequence of resistance training per se. We believe that it could be a result of better maintenance of lung function in a more consistent way during pulmonary rehabilitation. We did not perform a cumulative analysis of FVC due to a lack of suitable studies.

We found that the attrition rate was higher in the resistance-training group. However, there were no obvious differences between the 2 groups regarding the reasons for withdrawal. Moreover, there were no reports of adverse 
Resistance Training in Subjects With COPD

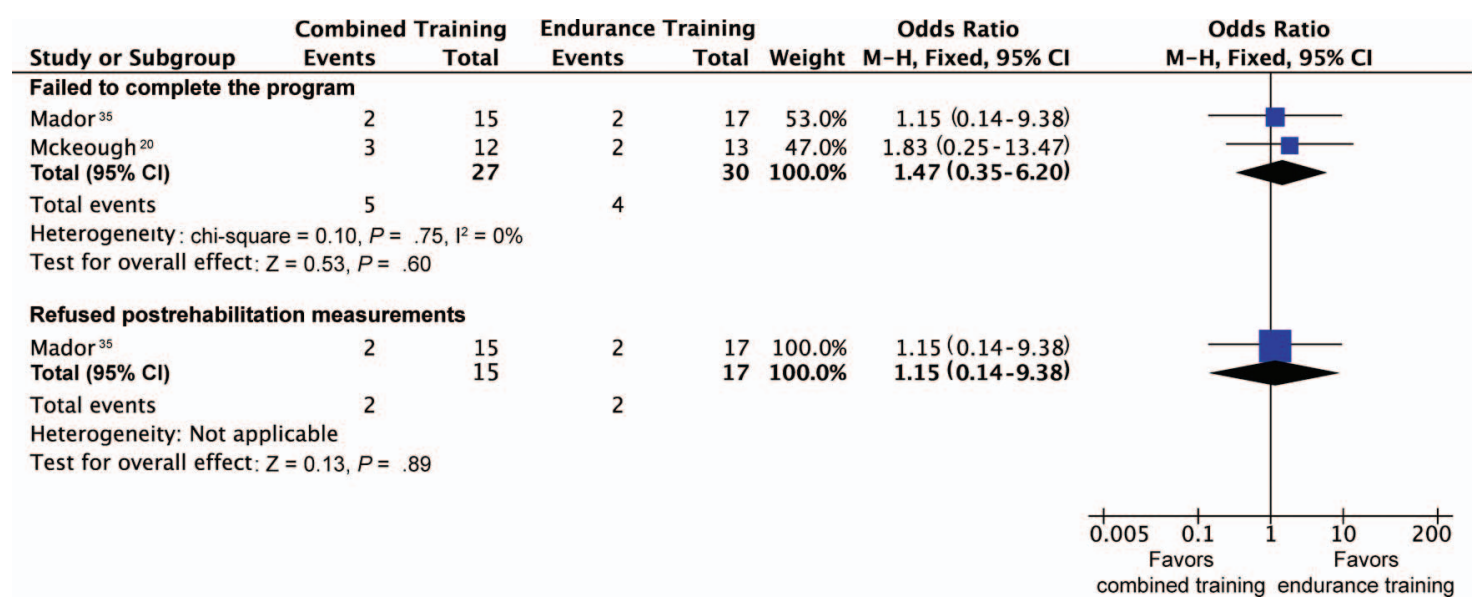

Fig. 10. Effects of combined resistance and endurance training vs endurance training alone on reasons for withdrawal. $\mathrm{M}-\mathrm{H}=\mathrm{Mantal}-$ Haenszel statistics.

events related to resistance-training intervention. Our results indicate that resistance training is a safe and tolerable modality of exercise training for patients with COPD.

The main strength of our study was inclusion of a large pool of subjects with COPD, allowing us to perform robust analysis of clinically relevant outcomes following resistance training versus no-exercise control or combined resistance and endurance training versus endurance training alone. The trials included in this analysis used almost identical designs with regard to inclusion and exclusion criteria, and the clinical characteristics of study populations were homogeneous. However, the results should be interpreted with caution because they might have been influenced by other factors. First, the duration of the resistancetraining intervention in most of included trials was too short to allow adequate evaluation of the long-term efficacy and exacerbations. Additional long-term studies are anticipated to answer this question. ${ }^{41}$ Second, the availability of outcome data suitable for meta-analysis was limited. For comparisons of resistance training versus nonexercise control or combined resistance and endurance training versus endurance training alone, there was a lack of sufficient number of studies reporting SGRQ and CRQ scores and lung function. Third, there is a potential risk of publication bias ${ }^{47}$ because negative findings are less likely to be published. We have not analyzed this aspect here. Fourth, none of the included studies reported the sample size calculation, although we were very rigorous in a thorough search of related publications. Based on the results of the sample size calculation, many of the included trials may have lacked sufficient sample size, which might be associated with bias. Fifth, the methodological quality of the 10 included RCTs was low to moderate. The reason may be explained by the fact that a double-blind design in studies on this topic may not be achievable. Despite this, we avoided including case-control studies, unlike several other related meta-analyses. Hence, our conclusions need further validation in large-sample studies. Finally, only 2 included trials performed intention-to-treat analysis. This suggested that most subjects included in our analysis were those who were able to or wanted to complete resistancetraining programs, which inevitably induced bias. The current limitations noted in many studies on the use of resistance training in patients with COPD, including ours, may encourage future improvements in the quality of related research.

Nevertheless, in our study, the clinical homogeneity of the trials resulted in statistical homogeneity for all outcome measures across the trials. Selection bias was minimized using a systematic search strategy, and we specified the inclusion and exclusion criteria. Furthermore, 2 reviewers independently evaluated the selected studies, and all authors consulted to reach consensus if necessary. Double counting of subjects from overlapping publications was avoided. Selective reporting of secondary end points in published manuscripts may also bias results. We minimized this bias by obtaining supplemental data for included studies.

\section{Conclusions}

In summary, this meta-analysis showed that dyspnea scale scores, skeletal muscle strength, and lung function improved following resistance training. Although skeletal muscle strength and quality of life improved following combined resistance and endurance training, they failed to translate into improved exercise capacity. Our results indicate that resistance training can be successfully performed alone or in conjunction with endurance training without increasing adverse events during pulmonary rehabilitation. Because of the limitations of this meta-analysis, we suggest further work to compare resistance training versus 


\section{Resistance Training in Subjects With COPD}

non-exercise control or combined resistance and endurance training versus endurance training alone. Larger, longer, multi-center, double-blind, parallel RCTs are needed to validate the long-term outcomes and safety of resistance-training programs for patients with COPD.

\section{ACKNOWLEDGMENTS}

We thank Guang-qiao Zeng MD (State Key Laboratory of Respiratory Disease, Guangzhou Medical University, Guangzhou, China) for writing assistance.

\section{REFERENCES}

1. Murray CJ, Lopez AD. Alternative projections of mortality and disability by cause 1990-2020: Global Burden of Disease Study. Lancet 1997;349(9064):1498-1504.

2. Gross NJ. Extrapulmonary effects of chronic obstructive pulmonary disease. Curr Opin Pulm Med 2001;7(2):84-92.

3. Kim HC, Mofarrahi M, Hussain SN. Skeletal muscle dysfunction in patients with chronic obstructive pulmonary disease. Int J Chron Obstruct Pulmon Dis 2008;3(4):637-658.

4. Decramer M, Gosselink R, Troosters T, Verschueren M, Evers G. Muscle weakness is related to utilization of health care resources in COPD patients. Eur Respir J 1997;10(2):417-423.

5. Swallow EB, Reyes D, Hopkinson NS, Man WD, Porcher R, Cetti EJ, et al. Quadriceps strength predicts mortality in patients with moderate to severe chronic obstructive pulmonary disease. Thorax 2007;62(2):115-120.

6. American Thoracic Society, European Respiratory Society. Skeletal muscle dysfunction in chronic obstructive pulmonary disease. Am J Respir Crit Care Med 1999;159(4 Pt 2):S1-S40.

7. Mador MJ, Bozkanat E. Skeletal muscle dysfunction in chronic obstructive pulmonary disease. Respir Res 2001;2(4):216-224.

8. Ortega F, Toral J, Cejudo P, Villagomez R, Sánchez H, Castillo J, Montemayor T. Comparison of effects of strength and endurance training in patients with chronic obstructive pulmonary disease. Am J Respir Crit Care Med 2002;166(5):669-674.

9. Houchen-Wolloff L, Sandland CJ, Harrison SL, Menon MK, Morgan MD, Steiner MC, Singh SJ. Ventilatory requirements of quadriceps resistance training in people with COPD and healthy controls. Int J Chron Obstruct Pulmon Dis 2014;9:589-595.

10. Probst VS, Troosters T, Pitta F, Decramer M, Gosselink R. Cardiopulmonary stress during exercise training in patients with COPD. Eur Respir J 2006;27(6):1110-1118.

11. Gloeckl R, Marinov B, Pitta F. Practical recommendations for exercise training in patients with COPD. Eur Respir Rev 2013;22(128): 178-186.

12. Covey MK, Collins EG, Reynertson SI, Dilling DF. Resistance training as a preconditioning strategy for enhancing aerobic exercise training outcomes in COPD. Respir Med 2014;108(8):1141-1152.

13. O'Shea SD, Taylor NF, Paratz J. Peripheral muscle strength training in COPD: a systematic review. Chest 2004;126(3):903-914.

14. O'Shea SD, Taylor NF, Paratz JD. Progressive resistance exercise improves muscle strength and may improve elements of performance of daily activities for people with COPD: a systematic review. Chest 2009;136(5):1269-1283.

15. Strasser B, Siebert U, Schobersberger W. Effects of resistance training on respiratory function in patients with chronic obstructive pulmonary disease: a systematic review and meta-analysis. Sleep Breath 2013;17(1):217-226.

16. Puhan MA, Schünemann HJ, Frey M, Scharplatz M, Bachmann LM. How should COPD patients exercise during respiratory rehabilita- tion? Comparison of exercise modalities and intensities to treat skeletal muscle dysfunction. Thorax 2005;60(5):367-375.

17. Benton MJ, Wagner CL. Effect of single-set resistance training on quality of life in COPD patients enrolled in pulmonary rehabilitation. Respir Care 2013;58(3):487-493.

18. Covey MK, McAuley E, Kapella MC, Collins EG, Alex CG, Berbaum ML, Larson JL. Upper-body resistance training and self-efficacy enhancement in COPD. J Pulm Respir Med 2012;(Suppl 9): 001

19. Janaudis-Ferreira T, Hill K, Goldstein RS, Robles-Ribeiro P, Beauchamp MK, Dolmage TE, et al. Resistance arm training in patients with COPD: a randomized controlled trial. Chest 2011;139(1):151158.

20. McKeough ZJ, Bye PT, Alison JA. Arm exercise training in chronic obstructive pulmonary disease: a randomised controlled trial. Chron Respir Dis 2012;9(3):153-162.

21. Nyberg A, Lindström B, Rickenlund A, Wadell K. Low-load/highrepetition elastic band resistance training in patients with COPD: a randomized, controlled, multicenter trial. Clin Respir J. 2014 [Epub ahead of print] doi: 10.1111/crj.12141.

22. Pereira AM, Santa-Clara H, Pereira E, Simões S, Remédios I, Cardoso J, et al. Impact of combined exercise on chronic obstructive pulmonary patients' state of health. Rev Port Pneumol 2010;16(5): 737-757.

23. Vonbank K, Strasser B, Mondrzyk J, Marzluf BA, Richter B, Losch $\mathrm{S}$, et al. Strength training increases maximum working capacity in patients with COPD—randomized clinical trial comparing three training modalities. Respir Med 2012;106(4):557-563.

24. Jadad AR, Moore RA, Carroll D, Jenkinson C, Reynolds DJ, Gavaghan DJ, McQuay HJ. Assessing the quality of reports of randomized clinical trials: is blinding necessary? Control Clin Trials 1996; 17(1):1-12

25. Zlowodzki M, Poolman RW, Kerkhoffs GM, Tornetta P 3rd, Bhandari M. How to interpret a meta-analysis and judge its value as a guide for clinical practice. Acta Orthop 2007;78(5):598-609.

26. Alexander JL, Phillips WT, Wagner CL. The effect of strength training on functional fitness in older patients with chronic lung disease enrolled in pulmonary rehabilitation. Rehabil Nurs 2008;33(3):9197.

27. Casaburi R, Bhasin S, Cosentino L, Porszasz J, Somfay A, Lewis $\mathrm{MI}$, et al. Effects of testosterone and resistance training in men with chronic obstructive pulmonary disease. Am J Respir Crit Care Med 2004;170(8):870-878.

28. Clark CJ, Cochrane LM, Mackay E, Paton B. Skeletal muscle strength and endurance in patients with mild COPD and the effects of weight training. Eur Respir J 2000;15(1):92-97.

29. Hoff J, Tjønna AE, Steinshamn S, Høydal M, Richardson RS, Helgerud J. Maximal strength training of the legs in COPD: a therapy for mechanical inefficiency. Med Sci Sports Exerc 2007;39(2):220-226.

30. Kongsgaard M, Backer V, Jørgensen K, Kjaer M, Beyer N. Heavy resistance training increases muscle size, strength and physical function in elderly male COPD patients-a pilot study. Respir Med 2004; 98(10):1000-1007.

31. Marrara KT, Marino DM, de Held PA, de Oliveira Junior AD, Jamami M, Di Lorenzo VA. Different physical therapy interventions on daily physical activities in chronic obstructive pulmonary disease. Respir Med 2008;102(4):505-511

32. O'Shea SD, Taylor NF, Paratz JD. A predominantly home-based progressive resistance exercise program increases knee extensor strength in the short-term in people with chronic obstructive pulmonary disease: a randomised controlled trial. Aust J Physiother 2007; 53(4):229-237.

33. Ries AL, Ellis B, Hawkins RW. Upper extremity exercise training in chronic obstructive pulmonary disease. Chest 1988;93(4):688-692. 


\section{Resistance Training in Subjects With COPD}

34. Simpson K, Killian K, McCartney N, Stubbing DG, Jones NL. Randomised controlled trial of weightlifting exercise in patients with chronic airflow limitation. Thorax 1992;47(2):70-75.

35. Mador MJ, Bozkanat E, Aggarwal A, Shaffer M, Kufel TJ. Endurance and strength training in patients with COPD. Chest 2004;125(6): 2036-2045.

36. Phillips WT, Benton MJ, Wagner CL, Riley C. The effect of single set resistance training on strength and functional fitness in pulmonary rehabilitation patients. J Cardiopulm Rehabil 2006;26(5):330-337.

37. Jaeschke R, Singer J, Guyatt GH. Measurement of health status: Ascertaining the minimal clinically important difference. Con Clin Trial 1989;10(4):407-415.

38. Marciniuk DD, Brooks D, Butcher S, Debigare R, Dechman G, Ford $\mathrm{G}$, et al. Optimizing pulmonary rehabilitation in chronic obstructive pulmonary disease-practical issues: a Canadian Thoracic Society Clinical Practice Guideline. Can Respir J 2010;17(4):159-168.

39. Spruit MA, Singh SJ, Garvey C, ZuWallack R, Nici L, Rochester C, et al. An official American Thoracic Society/European Respiratory Society statement: key concepts and advances in pulmonary rehabilitation. Am J Respir Crit Care Med 2013;188(8):e13-e64.

40. Bolton CE, Bevan-Smith EF, Blakey JD, Crowe P, Elkin SL, Garrod $\mathrm{R}$, et al. British Thoracic Society guideline on pulmonary rehabilitation in adults. Thorax 2013;68(Suppl 2):ii1-ii30.

41. Maltais F, Decramer M, Casaburi R, Barreiro E, Burelle Y, Debigaré $\mathrm{R}$, et al. An official American Thoracic Society/European Respira- tory Society statement: update on limb muscle dysfunction in chronic obstructive pulmonary disease. Am J Respir Crit Care Med 2014; 189(9):e15-e62.

42. Lewis MI, Fournier M, Storer TW, Bhasin S, Porszasz J, Ren SG, et al. Skeletal muscle adaptations to testosterone and resistance training in men with COPD. J Appl Physiol 2007;103(4):1299-1310.

43. Haas F, Salazar-Schicchi J, Axen K. Desensitization to dyspnea in chronic obstructive pulmonary disease. In: Casaburi R. Principles and practice of pulmonary rehabilitation. Philadelphia: WB Saunders; 1993; 241-251.

44. Gigliotti F, Coli C, Bianchi R, Romagnoli I, Lanini B, Binazzi B, Scano G. Exercise training improves exertional dyspnea in patients with COPD: evidence of the role of mechanical factors. Chest 2003; 123(6): 1794-1802.

45. Porszasz J, Emtner M, Goto S, Somfay A, Whipp BJ, Casaburi R. Exercise training decreases ventilatory requirements and exerciseinduced hyperinflation at submaximal intensities in patients with COPD. Chest 2005;128(4):2025-2034.

46. Casaburi R, Porszasz J, Burns MR, Carithers ER, Chang RS, Cooper CB. Physiologic benefits of exercise training in rehabilitation of patients with severe chronic obstructive pulmonary disease. Am J Respir Crit Care Med 1997;155(5):1541-1551.

47. Egger M, Smith GD. Meta-analysis bias in location and selection of studies. BMJ 1998;316(7124):61-66. 